\title{
Novel botanical therapeutic NB-02 effectively treats Alzheimer's neuropathophysiology in an APP/PS1 mouse model
}

\section{Yee Fun Lee}

Boston University

Amanda Lariviere

Massachusetts General Hospital

Alyssa N Russ

Massachusetts General Hospital

\section{Sang-Zin Choi}

NeuroBo

\section{Brian Bacskai}

Massachusetts General Hospital

Ksenia Kastanenka ( $\nabla$ kkastanenka@mgh.harvard.edu )

Massachusetts General Hospital https://orcid.org/0000-0002-3727-0317

\section{Research}

Keywords: Alzheimer's disease, multiphoton microscopy, therapeutic, in vivo, calcium imaging, DA-9803, NB-02

Posted Date: July 28th, 2020

DOI: https://doi.org/10.21203/rs.3.rs-48654/v1

License: (c) (i) This work is licensed under a Creative Commons Attribution 4.0 International License. Read Full License

Version of Record: A version of this preprint was published at eneuro on April 29th, 2021. See the published version at https://doi.org/10.1523/ENEURO.0389-20.2021. 
1 Novel botanical therapeutic NB-02 effectively treats Alzheimer's neuropathophysiology in

2 an animal model APP/PS1 mouse model

3

4 Yee Fun Lee ${ }^{1,2 \#}$, Amanda Lariviere ${ }^{2 \#}$, Alyssa N. Russ ${ }^{2}, \underline{\text { Sang-Zin } \text { Choi }^{3}}$, Brian J. Bacskai ${ }^{2}$, and

5 Ksenia V. Kastanenka ${ }^{2 *}$

6

7

8

9 Affiliation:

101 Department of Anatomy and Neurobiology, Boston University School of Medicine, Boston,

11 MA, 02118, USA.

122 Department of Neurology, MassGeneral Institute of Neurodegenerative Diseases,

13 Massachusetts General Hospital and Harvard Medical School, 114 Sixteenth St., Charlestown,

14 MA 02129.

153 NeuroBo Pharmaceuticals, Inc., 200 Berkeley St., Boston, MA 02116, USA.

16

17

18 \# These authors contributed equally

19

*Correspondence: kkastanenka@mgh.harvard.edu

20

21

22

23

24 


\section{Abstract}

28 Background

29 Alzheimer's disease $(A D)$ is an incurable neurodegenerative disorder and a major cause of

30 dementia. Some of the hallmarks of $A D$ include presence of amyloid plaques in brain

31 parenchyma, calcium dysregulation within individual neurons, and neuroinflammation. A

32 promising therapeutic would reverse or stymie these pathophysiologies in an animal model of

33 AD.

34 Methods

35 We tested the effect of NB-02, previously known as DA-9803, a novel multimodal therapeutic, on amyloid deposition, neuronal calcium regulation and neuroinflammation in 8-10 month old

37 APP mice, an animal model of AD.

38 Results

39 In vivo multiphoton microscopy revealed that 2 month-long administration of NB-02 halted

40 amyloid plaque deposition and cleared amyloid in the cortex. Post-mortem analysis verified NB-

41 02-dependent decrease in plaque deposition in the cortex as well as hippocampus.

42 Furthermore, drug treatment reversed neuronal calcium elevations, thus restoring neuronal

43 function. Finally, NB-02 transformed the morphology of astrocytes and microglia to a more

44 phagocytic state, affecting neuroinflammation.

45 Conclusions 
46 NB-02 was effective at reversing AD neuropathophysiology in an animal model. Therefore, in

47 addition to serving as a promising preventative agent, NB-02 holds potential as a treatment for $48 \quad A D$ in the clinic.

\section{Keywords}

51 Alzheimer's disease, multiphoton microscopy, therapeutic, in vivo, calcium imaging, DA-9803,

$52 \quad$ NB-02

\section{Background}

Alzheimer's disease $(A D)$ is a progressive neurodegenerative disorder currently without a cure[1,2]. It is characterized by presence of amyloid beta deposits in the brain parenchyma[3,4]. Circuit dysfunctions underlying cognitive and memory deficits may stem from amyloid betadependent calcium dyshomeostasis resulting in elevations of baseline calcium within individual neurons[5,6]. In addition to deposition of amyloid plaques and disruption of calcium homeostasis, AD is characterized by substantial inflammatory response[7-9], manifesting in increased expression of inflammatory markers in non-neuronal cells, such as astrocytes and microglia[10]. Restoration of neuronal calcium to control levels and normalization of neuroinflammatory response would indicate treatment efficacy[11,12].

NB-02, previously known as DA-9803, is a novel proprietary botanical cocktail containing extracts from Morus alba L. and Poria cocos[13]. The extract is reproducibly prepared according to a standardized recipe. Currently in preclinical development by NeuroBo Pharmaceuticals, it is a multimodal therapeutic that has been shown to prevent deposition of amyloid plaques in young APP/PS1[14] mice, an animal model of Alzheimer's disease[13]. In addition, it was 

preventative therapeutic for AD. However, what is its treatment potential?

effective at preventing neuronal calcium elevations, thus maintaining circuit integrity in these mice. Finally, it transformed astrocytes and microglia, allowing these non-neuronal cells to restore neuronal calcium homeostasis, and modify neuroinflammation. Interestingly, NB-02 in the clinic.

\section{Methods}

\section{Animals and surgery}

Transgenic APPswe/PS1dE9 mice were used (10 males, 11 females; B6:C3-Tg(APPswe, with Massachusetts General Hospital Animal Care and Use Committee as well as NIH guidelines for the use of laboratory animals. over the right hemisphere[15] on the same day starting at 7 months of age similar to earlier assume more phagocytic morphology. Thus NB-02 is an ideal candidate to enter the clinic as a

To address this question, 8 month old APP/PS1 mice were treated with daily gavage of $300 \underline{100}$ mg/kg NB-02 for 2 months. We tested the therapeutic's propensity to slow amyloid deposition, halted amyloid deposition and cleared some amyloid plaques in cortices of drug-treated animals compared to vehicle-treated mice. Furthermore, it restored calcium homeostasis in neurons that started out with elevated baseline calcium levels prior to treatment onset. Moreover, it modified morphology of astrocytes and microglia, thus affecting neuroinflammation. Theretofore, NB-02 had a restorative effect on neuronal and non-neuronal cells in an AD mouse model. Thus, it has great potential to restore healthy circuit function and thereby slow cognitive and memory decline PSEN1dE9)85Dbo/Mmjax background). These animals overexpress the Swedish mutation in the human APP gene and deltaE9 mutation in the Presenilin gene[14]. All the studies complied

APP/PS1 mice underwent intracortical virus injections followed by cranial window installations 
reports[11,13,16,17]. Following anesthesia induction, animals were secured in a stereotaxic apparatus. After disinfection of skin, incision was made, skull was cleared and burr holes were drilled through the skull. Using a $10 \mu$ Hamilton syringe, $3 \mu$ l of AAV8-YellowCameleon 3.6 (YC3.6) (U.Penn Vector Core) was injected in the cortex with the following coordinates: anteriorposterior -1.5 , medial-lateral -2 , dorsal-ventral -0.8 at the rate of $130 \mathrm{nl} / \mathrm{min}$. Thus, excitatory neurons in right somatosensory cortex were targeted. YC3.6 is a genetically encoded calcium indicator (GECl) that allows determination of absolute intracellular calcium concentration based on its ratio of YFP/CFP in individual cells[18]. Viral injections were directly followed by craniotomies over the injection sites. Cranial windows with $5 \mathrm{~mm}$ diameter were fixed with a mixture of dental cement and crazy glue. Body temperature of each animal was maintained through the entire surgical procedure and during the recovery from anesthesia. YC3.6 was allowed to express for a month prior to imaging with multiphoton microscopy. This also allowed the inflammatory response associated with craniotomies to subside. Drug treatment and multiphoton imaging commenced when animals reached 8 months of age.

Therapeutic treatment

APP/PS1 mice were randomly assigned to the vehicle or the NB-02 treatment conditions. Vehicle consisted of hydroxypropyl methyl cellulose (HPMC) dissolved in water. NB-02 drug consisted of NB-02 mixed with vehicle solution. Drug or vehicle treatment was initiated subsequent to the baseline imaging session. Treatment was administered to the animals via

112 daily gavage with $300 \mu \mathrm{l}$ of $300 \underline{100} \mathrm{mg} / \mathrm{kg} \mathrm{NB}-02$ or vehicle for the duration of 2 months. Drug 113 treatment had no significant effect on animal attrition (data not shown). Treatment continued 114 while the animals were imaged at 1 month and 2 month time-points after treatment onset. 115 Subsequent to the last imaging session, animals received their last gavage treatment, after 116 which mice were euthanized and perfused transcardially with PBS. Their brains were isolated 
117 and processed for immunohistochemistry or biochemistry. 10 animals were treated with vehicle,

11811 animals were treated with NB-02, totaling 21 mice.

\section{Imaging using multiphoton microscopy}

120 Prior to the baseline imaging session, animals received intraperitoneal injections of $4 \mathrm{mg} / \mathrm{kg}$

121 methoxy-XO4, which crosses the blood-brain barrier and binds to amyloid plaques allowing

122 visualization by multiphoton microscopy[19-21]. Animals were anesthetized with $2 \%$ isoflurane

123 and secured into a stereotactic stage. Intravenous administration of Texas Red dextran into the

124 retro-orbital sinus provided a fluorescent angiogram. Multiphoton microscopy allowed imaging of

125 the angiogram, amyloid plaques and YC3.6-expressing neurons using an Olympus Fluoview

126 1000MPE mounted on an Olympus BX61WI upright microscope. Same fields of view were

127 identified during all 3 imaging sessions (baseline, 1 month, 2 months) using the landmarks

128 present in the angiogram collected during the baseline imaging session. Images were acquired

129 using a $25 \times$ water immersion objective (NA = 1.05). A mode-locked titanium:sapphire laser

130 (MaiTai; Spectra-Physics, Fremont, CA, USA) generated two-photon fluorescence with either

$131800 \mathrm{~nm}$ or $860 \mathrm{~nm}$ excitation. Amyloid plaques were imaged using $800 \mathrm{~nm}$ excitation at $1 \times$

132 zoom. Z stacks were acquired at $5 \mu \mathrm{m}$ step-size. YC3.6 was imaged using $860 \mathrm{~nm}$ excitation at

$1332 \times$, and $5 \times$ zoom. These $z$ stacks were acquired at $1 \mu \mathrm{m}$ step-size. Laser power was limited

134 below $30 \mathrm{~mW}$ to prevent phototoxicity. Animals body temperature was maintained with a heating

135 pad throughout imaging. Methoxy-XO4 was re-administered prior to each imaging session to

136 identify newly appeared amyloid plaques.

137 At the end of each imaging session, animals were allowed to recover from anesthesia while

138 their body temperature was maintained with a heating pad. At the end of the last imaging

139 session, mice received their last gavage treatment and their CSF was collected (see below).

140 Then mice were euthanized using $\mathrm{CO}_{2}$. Their bodies were perfused with PBS, and brains 
141 isolated. Left hemibrains (that had not contained cranial windows) were fixed with 4\%

142 paraformaldehyde and cryoprotected with 15\% glycerol overnight. Optimal Cutting Temperature

143 compound (OCT) was used to freeze the hemibrains, which were subsequently cut into $20-\mu \mathrm{m}$

144 coronal sections on a cryostat, and mounted onto slides for immunohistochemical processing.

145 Right hemibrains (that had contained cranial windows) were flash frozen in liquid nitrogen and

146 subsequently processed for biochemistry.

\section{Cerebrospinal fluid (CSF) collection}

148 Prior to euthanasia, each animal was anesthetized with $2 \%$ Isoflurane and secured into a

149 stereotaxic apparatus. Cisterna magna containing CSF was identified. Dura was punctured with 150 a 30 gauge needle. CSF was collected using P20 pipette using dissection microscope. CSF was 151 stored in low protein binding tubes in $-80^{\circ} \mathrm{C}$ before use.

\section{Data analysis}

153 Analysis was performed using ImageJ software (http://rsbweb.nih.gov/ij/) to determine amyloid 154 plaque numbers, amyloid plaque burden, and resting calcium levels within individual neuronal 155 processes, or neurites. Since the same fields of view were imaged over time, the same amyloid 156 plaques and neuronal processes could be tracked longitudinally to determine amyloid plaque 157 burden and intracellular calcium changes in each individual neuronal process. To determine 158 amyloid plaque numbers and amyloid plaque burden, each z-stack was processed into a 159 maximum intensity projection. Amyloid plaques were counted manually in each projected image 160 to determine the amyloid plaque number. Each projected image was thresholded, segmented, 161 and the percentage area occupied by amyloid was measured to calculate the amyloid burden.

162 The signal from the amyloid present in blood vessels, cerebral amyloid angiopathy, was 163 excluded from analysis. 
164 The images containing neuronal processes expressing YC3.6 were also analyzed using

165 ImageJ. YC3.6 is a FRET probe, where a donor, cyan fluorescent protein (CFP), and an

166 acceptor, yellow fluorescent protein (YFP) are connected by a linker[18]. Intracellular calcium

167 concentrations were determined from the ratio of YFP to CFP. The higher the calcium

168 concentration, the greater the YFP to CFP ratio. The background for each channel was

169 calculated by the mode of the intensities of the last slice of each volume. That value was

170 subtracted from its channel. A median filter with a radius of 2 was applied to the fluorescence

171 intensities. YFP was divided by CFP, thus creating a ratio image that could be converted to

172 absolute calcium concentration. Neurons were identified and their processes selected manually

173 as regions of interest (ROIs) using the 'free hand' tool on ImageJ in the YFP images. These

174 neuronal ROls were then exported to the ratio images and the YFP/CFP ratios calculated. The

175 relative change in YFP/CFP ratio $\left(\Delta R / R_{i}\right)$ was calculated by tracking the same neurites

176 throughout all three imaging sessions. YFP/CFP ratios were converted to $\left[\mathrm{Ca}^{2+}\right]$ with standard

177 equations using the in-situ $K_{d}$ and Hill coefficient for YC3.6 determined earlier[16]. Matlab was

178 used to create pseudocolored images based on the calcium concentration using the

179 empirical $R_{\min }$ and $R_{\max }$. The ratio values were used to determine the hue and saturation (color)

180 and the brightness values were used to assign the value (intensity) in the pseudocolored

181 images.

182 Cell culture

183 KCLB No. 22266 SH-SY5Y cell line (human neuroblastoma cell line, passage no. 64) was

184 purchased from the Korean Cell Line Bank. Cells were maintained in a $5 \% \mathrm{CO}_{2}$ incubator at

$185 \underline{37^{\circ} \mathrm{C}}$ in DMEM/F12 media (Gibco) containing 10\% FBS (fetal bovine serum) and $1 \% \mathrm{P} / \mathrm{S}$

186 (penicillin/streptomycin). 48 hours after seeding (6-well plate: 2x105 cells, 96-well plate: 1 x104

187 cells), it was maintained in DMEM/F12 medium containing 3\% FBS and retinoic acid (final conc.

$188 \quad 10 \mu \mathrm{M})$ for 5 days. 
$190 \quad$ MTT assay

191 Cell cultures were exposed to $1 \mu \mathrm{M}$ oligomeric Aß1-42 (Sigma-Aldrich) for 24 hours followed by

192 treatment with a single concentration of -NB-02 for 48 hours. The following concentrations of

$193 \quad$ NB-02 were used: $0.1,0.3,1,3,10,30,100,300 \mu \mathrm{g} / \mathrm{ml}$. Alternatively to NB-02, cell cultures

194 were treated with donepezil (donepezil HCl, Waco) in presence of oligomeric Aß1-42. The

195 following concentrations of donepezil were used: 0.1, 1, $10 \mu \mathrm{M}$. Serotonergic receptor blocker

196 Lu AE58054 (Lu AE58054 Hydrochloride, 1, $10 \mu \mathrm{M}$, Chem Scene) was used in presence of

197 oligomeric Aß1-42 and NB-02. Then cell cultures were treated with $50 \mu \mathrm{l}$ of MTT (3-(4,5-

198 Dimethyl-2-thiazolyl)-2,5- diphenyl-2H-tetrazolium bromide, Sigma Aldrich) for 4 hours. After

199 removing the supernatant, $200 \mu$ l of DMSO (Sigma-Aldrich) was added and shaken lightly. The

200 absorbance was measured with a microplate spectrophotometer at $595 \mathrm{~nm}$.

201

202 Acetylcholinesterase activity assay

203 Acetylcholinesterase activity was determined using the Ellman's method [22]. Subsequent to 24

204 hour-pretreatment with Aß1-42 followed by 48 hour treatment with each concentration of NB-02.

205 cells were harvested in RIPA buffer. Cells were centrifuged at 13,000 rpm for 15 minutes to

206 obtain the supernatant. Proteins were quantified using the Bio-rad protein assay kit (Bio-rad

207 Labs). - Acetylcholinesterase activity was measured using the Abcam assay kit (Abcam). Each

208 sample was mixed with DTNB, acetylthiocholine and assay buffer in a 96-well microplate,

209 followed by incubation for 10 minutes at $37^{\circ} \mathrm{C}$. Acetylcholinesterase activity was measured using

210 the microplate spectrophotometer at $405 \mathrm{~nm}$ absorbance. The enzyme activation was presented

211 in $\mathrm{mU} / \mathrm{mg}$ protein.

212

$213 \quad$ NGF assay 
214 Cells were treated with NB-02 for 48 hours. Then cells were incubated with lysis buffer (100 mM

215 of Tris/ $\mathrm{HCl}$ pH.7.0 containing 2\% bovine serum albumin, $1 \mathrm{M} \mathrm{NaCl}, 4 \mathrm{mM}$ EDTA.Na2, 2\% Triton

$216 \underline{\mathrm{X}-100,0.1 \% \text { sodium azide, } 5 \mu \mathrm{gg} / \mathrm{ml} \text { of aprotinin protease inhibitor, } 0.5 \mu \mathrm{g} / \mathrm{ml} \text { of antipain, } 157}$

$217 \mu \mathrm{gg} / \mathrm{ml}$ of benzamide, $0.1 \mu \mathrm{g} / \mathrm{ml}$ of pepstatin A and $17 \mu \mathrm{g} / \mathrm{ml}$ of phenylmethyl-sulphonyl fluoride)

218 and harvested. Samples were centrifuged at $13,000 \mathrm{rpm}$ for 15 minutes to obtain the

219 supernatant. Proteins were quantified using the Bio-rad protein assay kit (Bio-rad Labs). Protein

220 NGF levels were measured using NGF ELISA kit (-Abcam). The microplate spectrophotometer

221 absorbance was measured at $450 \mathrm{~nm}$-. The quantity of NGF was presented in $\mathrm{pg} / \mathrm{mg}$ protein.

\section{Immunohistochemistry and image analysis}

$22420-\mu \mathrm{m}$ coronal sections of mouse brains were subjected to antigen retrieval in citrate buffer. The

225 sections were then permeabilized using Triton X-100, blocked with normal goat serum (NGS),

226 and incubated with the following primary antibodies: 6E10 (monoclonal 6E10, 1:500; Covance),

227 glial fibrillary acidic protein (GFAP) (mouse monocolonal anti-GFAP, 1:200; Thermo Scientific),

228 or lba-1 (rabbit monoclonal anti-lba1, 1:200; ab178846) at $4^{\circ} \mathrm{C}$ overnight. This was followed by

229 incubation with the respective secondary antibodies (1:500) for 1 hour at room temperature. The

230 slides were then mounted with Vectashield antifade mounting media (Vector Laboratories)

231 either with or without DAPI.

232 For amyloid plaque burden analysis, 20- $\mu \mathrm{m}$ sections with methoxy-XO4-labeled plaques and ex

233 vivo 6 E10 immunostaining were imaged using an inverted Zeiss microscope with a 20x

234 objective. Images were thresholded and amyloid burden was calculated as a percentage of the

235 cortical or hippocampal area.

236 Coronal hemibrain sections stained with GFAP or lba-1 were imaged using an inverted Zeiss

237 microscope at 10x for the purpose of glial cell counts. Regions of interest (ROls) were drawn of 
238 the whole hippocampus or a randomly selected section of cortex using the ImageJ "free hand"

239 or "rectangle" tools respectively. Cells within the ROI were manually counted while blinded to

240 condition to minimize bias. Representative images were taken with 20x objective.

241 GFAP and Iba-1 stained sections were also imaged using an inverted Olympus confocal

242 microscope with a 40x objective for the purpose of morphology analysis. ImageJ was used to

243 analyze markers of cell morphology such as process length, cell body diameter, soma area, and

244 shape. Process length was measured as distance from the edge of the soma to the end of the

245 process. Cell body diameter was measured using the Feret's diameter, the maximum caliper of

246 the cell.

\section{Amyloid-beta ELISAs}

248 Total soluble amyloid beta 40 and 42 levels were measured using the amyloid beta 40 and 42

249 sandwich ELISA kits (Wako). Each flash-frozen hemibrain was homogenized in ice-cold tris-

250 buffered saline (TBS) in presence of phosphatase inhibitor and centrifuged at 25500 rpm for 30

251 min at $4{ }^{\circ} \mathrm{C}$. Supernatants containing soluble amyloid beta were collected and subjected to

252 ELISAs using the manufacturer's instructions. Amyloid beta 40 and 42 levels were determined

253 using Amyloid beta 40 and 42 standard calibration curves. Protein levels were normalized to wet

254 brain weights. Similarly, Amyloid beta 40 and 42 levels were determined in CSF samples.

\section{Statistics}

256 GraphPad 5.0 was used to run statistical analyses. Data was represented as mean \pm SEM.

257 Datasets were tested for normality using the Shapiro-Wilk normality test or Kolmogorov-Smirnov

258 test. Normally distributed datasets were subjected to parametric tests, such as t-tests or

259 ANOVAs. Nonparametric datasets were compared using nonparametric tests, such as Mann

260 Whitney or Kruskal-Wallis tests. Repeated measures datasets were compared using 
261

262 significant.

264 Results

\section{NB-02 is effective at halting amyloid plaque deposition in old APP/PS1 mice}

266 NB-02 is a multimodal botanical cocktail containing extracts from Morus alba L. and Poria

267 cocos. To test its propensity for treatment of AD pathology in vivo, 8 month old APP/PS1 mice 268 were subjected to daily gavage treatments with 300100 mg/kg NB-02 dose or vehicle cocktail 269 (Fig. 1a). Methoxy-X04[19,23] was administered intraperitoneally, crossed blood-brain barrier, 270 and bound to amyloid deposits to allow visualization of amyloid plaques within the same fields of

271 view of cranial windows using high-resolution multiphoton microscopy over the course of

272 treatment[23-25]. A fluorescent angiogram was acquired during baseline imaging session after

273 injection of Texas Red Dextran to allow visualization of the field of view and increase reliability

274 of finding the same fields of view during subsequent imaging sessions (Fig. 1b, e). Daily gavage

275 treatment was initiated on the same day subsequent to baseline imaging session, starting at 8

276 months of age, and multiphoton imaging was repeated 1 month and 2 months after treatment

277 onset.

278 Animals randomly assigned to vehicle and drug treatment conditions started out with similar 279 numbers of plaques in somatosensory cortex (154 \pm 9 plaques $/ \mathrm{mm}^{3}$ across 62 z-stacks in 10 280 mice treated with vehicle, $159 \pm 11$ plaques $/ \mathrm{mm}^{3}$ across 63 -stacks in 11 mice treated with NB-

281 02, mean \pm SEM, Mann Whitney test, n.s.). Vehicle-treated animals exhibited increases in the 282 number of amyloid deposits within the cortical fields of view over time (Fig. 1d compared to 1c, 283 yellow arrow). Amyloid plaque numbers increased to $200 \pm 12$ plaques $/ \mathrm{mm}^{3}$ in vehicle-treated 284 animals 1 month after treatment onset, significantly different from baseline (Fig. 1h, mean \pm SEM, 
Wilcoxon matched-pairs signed rank test $p<0.001$ ). Plaque deposition again increased to $243 \pm 16$ plaques $/ \mathrm{mm}^{3} 2$ months after treatment onset, significantly different from baseline (Fig. 1h, mean $\pm S E M$, Wilcoxon matched-pairs signed rank test $p<0.001)$. Interestingly, NB-02 halted amyloid depositions and resulted in clearance of amyloid plaques in APP/PS1 mice (Fig. $1 \mathrm{~g}$ compared to 1f). NB-02-treated animals experienced a decrease in plaque numbers per cortical volume at 1 month after treatment onset (Fig. $1 \mathrm{~h}, 146 \pm 11$ plaques $/ \mathrm{mm}^{3}$ mean $\pm S E M$, Wilcoxon matched-pairs signed rank test $\mathrm{p}<0.05)$. By 2 months after treatment onset, the number of amyloid plaques decreased to $139 \pm 14$ plaques $/ \mathrm{mm}^{3}$ (Wilcoxon matched-pairs signed rank test $p<0.01)$. At the end of treatment period, average plaque number was significantly lower in NB02-treated animals compared to those treated with vehicle (Fig. 1i, $243 \pm 16$ plaques $/ \mathrm{mm}^{3}$ for vehicle, $139 \pm 14$ plaques $/ \mathrm{mm}^{3}$ for NB-02, mean $\pm S E M$, Mann Whitney test $p<0.001$ ).

Amyloid plaque burden analysis was performed, taking into account the number and size of plaques. At baseline, vehicle-treated animals exhibited $0.98 \pm 0.05 \%$ burden (mean $\pm S E M$ ), while NB-02 treated mice had $1.04 \pm 0.06 \%$ burden (mean \pm SEM, Mann Whitney test, n.s.). Amyloid plaque burden increased to $1.33 \pm 0.07 \%$ in vehicle-treated animals 1 month after treatment onset, significantly higher compared to baseline (Fig. 1I, mean \pm SEM, Wilcoxon matched-pairs signed rank test $p<0.001)$. By 2 months after treatment onset, amyloid plaque burden increased higher to $1.69 \pm 0.11 \%$, significantly different from baseline (Fig. 1I, mean \pm SEM, Wilcoxon matched-pairs signed rank test $\mathrm{p}<0.001)$. Complementary to plaque number findings, amyloid plaque burden decreased to $1.0 \pm 0.05 \%$ in NB-02-treated mice after 1 month (Fig. 11, mean $\pm S E M$, Wilcoxon matched-pairs signed rank test $p<0.01$ ) and then decreased further to $0.82 \pm 0.06 \%$ after 2 months of treatment with NB-02 (Fig. 1l, mean \pm SEM, Wilcoxon matchedpairs signed rank test $p<0.001$ ). Thus, by the end of treatment, vehicle-treated mice had substantially higher amyloid burden $(1.69 \pm 0.11 \%)$ compared to NB-02 treated mice $(0.82 \pm 0.06 \%)$ (Fig. $1 \mathrm{~m}$, mean \pm SEM, Mann Whitney test $p<0.001)$. 
These findings suggest that while vehicle treatment failed to prevent deposition of new plaques,

311 NB-02 treatment led to plaque clearance as imaged in vivo (Fig. 1j, Mann Whitney test,

$312 \mathrm{p}<0.001, \mathrm{n}=46$ comparisons in 10 mice for vehicle, $\mathrm{n}=48$ comparisons in 11 mice for NB-02).

313 Amyloid plaque burden analysis reflected this conclusion (Fig. 1n, Mann Whitney test, $p<0.001$ ).

314 Even though the plaque size was comparable in vehicle and drug conditions (Fig. 1k), treatment

315 with NB-02 led to decreases in intensity of individual plaques (Fig. 10, Mann Whitney test,

$316 \mathrm{p}<0.001)$. Since we had verified that neither NB-02 nor vehicle interfered with methoxy-X04

317 binding to individual plaques[13], decreases in plaque intensity signaled bona fide plaque

318 clearance in NB-02 condition.

319 As multiphoton microscopy allowed imaging of small cortical regions, we set out to verify the 320 above findings in larger cortical regions using coronal cross-sections of hemibrains processed 321 post-mortem at the end of treatment. Brain sections were immunostained with anti-amyloid beta 322 antibody 6E10. While methoxy-X04 labelled dense cores, 6E10 decorated the periphery of 323 amyloid plaques as well (Fig. 2a-f). Vehicle-treated brains exhibited high amyloid plaque load, 324 evident with 6E10 immunoreactivity and methoxy-X04 labeling (Fig. 2a, b, c). Intriguingly, NB-02 325 treatment resulted in decreased amyloid deposition (Fig. 2d, e, f). 6E10 immunoreactivity 326 suggested that amyloid plaque burden was significantly lower as a result of NB-02 treatment 327 compared to vehicle (Fig. $2 g$, Mann Whitney test, $p<0.01, n=21$ sections in 8 mice/vehicle; $n=25$ 328 sections in 8 mice/NB-02). Similarly, methoxy-X04 labeling indicated lower plaque burden at the 329 end of NB-02 treatment (Fig. 2h, Mann Whitney test, $\mathrm{p}<0.01$ )

330 Amyloid plaque burden was also determined in hippocampi of treated mice post-mortem (Fig. 331 3). There, $6 \mathrm{E} 10$ detected amyloid on the periphery of plaques in addition to their centers, while 332 methoxy-X04 identified dense cores exclusively (Fig. 3a-f). Similar to the data in the cortex, NB33302 treatment resulted in decreased amyloid plaque burden as assessed by $6 \mathrm{E} 10$ 334 immunoreactivity (Fig. 3g Mann Whitney test, $p<0.05, n=24$ sections in 8 mice/vehicle; $n=23$ 
sections in 8 mice/NB-02) or methoxy-X04 labeling (Fig. 3h Mann Whitney test, $p<0.01)$. We had previously verified that neither NB-02 nor vehicle interfered with methoxy-X04 binding to amyloid plaques[13]. Thus, amyloid plaque burden analysis performed post-mortem agreed with the in vivo data (Fig. 1), where treatment with NB-02 resulted in amyloid plaque clearance in an animal model of amyloidosis.

\section{NB-02 treatment does not alter soluble amyloid beta levels measured with ELISAs in CSF or TBS-soluble brain fractions}

To determine whether treatment with NB-02 altered soluble amyloid beta levels, cerebrospinal fluid (CSF) samples were collected from cisterna magna of APP/PS1 mice at the end of the treatment with the drug or the vehicle. ELISAs specific for amyloid beta 40 or 42 were used to measure protein levels. Interestingly, CSF amyloid beta 40 levels showed a trend toward an increase in animals treated with NB-02, however failed to reach statistical significance (Fig. S1a). Similarly, CSF amyloid beta 42 levels measured after NB-02 treatment also tended to increase, however these levels were not statistically different from those after vehicle treatment (Fig. S1b).

We also measured TBS-soluble amyloid beta 40 and 42 levels in brains of APP/PS1 mice treated with NB-02 or vehicle. Amyloid beta 40 levels in TBS soluble fractions trended up in NB02 condition compared to vehicle (Fig. S1c), not reaching significance. Furthermore, TBSsoluble amyloid beta 42 levels were not significantly different between the two conditions, yet further supported the trend up in NB-02-treated brains (Fig. S1d). Thus, the ELISA results suggest that neither soluble amyloid beta 40 nor 42 levels were significantly altered by NB-02 treatment, albeit showing an upward trend. 


\section{NB-02 restores neuronal calcium homeostasis}

360 To determine whether NB-02 treatment has a direct effect on neuronal health, we assessed NB-

361 02's propensity to restore calcium homeostasis within neuronal processes, neurites. Animal

362 models of amyloidosis contain a small cortical neuronal population that is vulnerable to amyloid

363 beta-dependent calcium dysregulation. This results in baseline calcium elevations within these

364 neurons[26]. Therefore, restoration of neuronal calcium to control levels would serve as a

365 functional indicator of treatment efficacy. Hence, we determined whether gavage treatment with

366 NB-02 would alter neuronal calcium. A genetically encoded calcium indicator, YellowCameleon

$3673.6[18]$ was virally expressed in cortical neurons (Fig. 1a) to calculate absolute baseline calcium

368 concentration within each neuron expressing the calcium reporter. Healthy neuronal function is

369 maintained at $\sim 100 \mathrm{nM}$ intracellular calcium. Amyloid beta present in animal models of

370 amyloidosis, such as APP/PS1 mice used here, leads to calcium elevations within a fraction of

371 cortical neurons (Fig. 4a). Calcium overload was defined as YFP/CFP ratio that was 2 standard

372 deviations above the mean in healthy wildtype mice, and calculated to be $>1.79$. As anticipated,

373 we saw $11 \%$ of neurons exhibiting calcium overload prior to vehicle treatment onset (Fig. 4c

374 blue bar in red rectangle) and 9\% of neurons exhibiting calcium overload before DA-02

375 treatment commenced (Fig. 4d blue bar in red rectangle), while the majority of neurons

376 exhibited healthy calcium levels, YFP/CFP $<1.79$. As vehicle treatment progressed the

377 percentage of neurons exhibiting calcium overload increased, reaching $30 \%$ by the end of

378 treatment (Fig. 4c). Surprisingly, there was a substantial decrease in the percentage of neurons

379 with calcium overload 1 month after NB-02 treatment onset (Fig. 4d purple bar in red rectangle).

380 The low percentage of neurons with calcium overload was maintained at 2 months of treatment

381 (Fig. 4b, d red bar in red rectangle). At the end of treatment, the percentage of neurites with

382 calcium overload was substantially lower in the NB-02-treated cortices $(3.32 \pm 3 \%)$ compared to

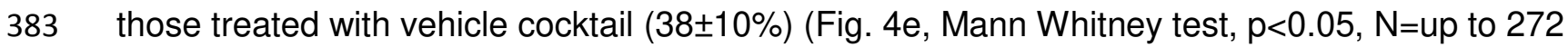


neurites in 4 mice/vehicle; $\mathrm{N}=u$ up to 368 neurites in 6 mice/NB-02). Thus, NB-02 treatment was effective at restoring calcium homeostasis and maintaining optimal intracellular calcium levels for healthy neuronal function.

\section{NB-02 treatment transforms morphology of glial cells}

Neuroinflammation is one of the major hallmarks of AD[27-29]. Thus, the effects of NB-02 on non-neuronal cells such as astrocytes and microglia were analyzed using immunohistochemistry post mortem. The number of cells positive for glial fibrillary acidic protein (GFAP)[30,31] or ionized calcium binding adaptor molecule 1 (Iba-1) were counted in the cortices and hippocampi to determine differences in relative cell numbers at the end of treatment. In addition, morphological analyses were performed by measuring the length of processes and the size of somas. Upon analyzing cell counts, no significant differences were found in the number of GFAP positive astrocytes between vehicle and NB-02 treated animals in either cortex (Fig. 5a-c, Mann-Whitney test, n.s; $\mathrm{N}=8$ sections in 8 mice/vehicle, $\mathrm{N}=7$ sections in 7 mice/NB-02) or hippocampus (Fig. 5d-f, Mann-Whitney test, n.s; N=7 sections in 7 mice/vehicle, $\mathrm{N}=7$ sections in 7 mice/NB-02). Similarly, there were no significant differences in Iba-1 positive cell numbers across condition for either cortex (Fig. 6a-c, Mann-Whitney test, n.s; $\mathrm{N}=8$ sections in 8 mice/vehicle, $\mathrm{N}=8$ sections in 8 mice/NB-02) or hippocampus (Fig. 6d-f, Mann-Whitney test, n.s; $\mathrm{N}=8$ sections in mice/vehicle, $\mathrm{N}=5$ sections in 5 mice/NB-02).

While no significant differences were found in the numbers of reactive cells, morphological analyses revealed differences across conditions for both astrocytes and microglia. Astrocytic morphology was altered as a result of NB-02 treatment, as evidenced by shorter processes and larger cell bodies (Fig. 5h, i, j; t-tests, $\mathrm{p}<0.0001$ for processes and cell body diameter; $\mathrm{N}=50$ cells, 100 processes in 5 mice/NB-02). In contrast vehicle-treated astrocytes maintained their 
408 long processes and small cell bodies (Fig. $5 \mathrm{~g}$, i, j; N=50 cells, 100 processes in 5 mice/vehicle).

409 Similarly, morphology of microglia was changed after NB-02 treatment also evidenced by

410 shorter processes and larger cell bodies, both by diameter and area (Fig. 6h-I; Mann-Whitney

411 test, $\mathrm{p}<0.0001$ for all comparisons; $\mathrm{N}=414$ cells, 1080 processes in 8 mice/NB-02). Microglia in

412 animals treated with vehicle possessed long processes and small cell bodies (Fig. 6g, i-I; N=475

413 cells, 1040 processes in 8 mice/vehicle). To confirm that the significant differences across

414 condition were not simply due to the large numbers of cells being analyzed, the same statistical

415 analyses were performed with cells grouped by mouse, and the results were verified (Fig. S2a-

416 e). Thus, in addition to having an effect on neuronal cells, NB-02 had an effect on non-neuronal

417 cells such as astrocytes and microglia.

$418 \quad$ NB-02 is a multimodal therapeutic with multiple mechanisms of action

419 To gain insight into the mechanism(s) of action of NB-02, we assessed the effects of NB-02 in

420 cell culture. Alzheimer's disease is a neurodegenerative disorder, thus we tested the

421 neuroprotective effects of NB-02. Nerve growth factor (NGF) is a neurotrophin that plays a

422 neuroprotective role by promoting maintenance, survival and plasticity of neurons. NGF deficits

423 induce neuronal apoptosis, death and dysfunction as a result of $A \beta$-induced toxicity in the

424 brain[32]. Through neurotrophic tyrosine receptor kinases (NTRKs), NGF has also been shown

425 to prevent amyloid precursor protein (APP) cleavage by $\beta$-secretase 1 (BACE1) [33]. Finally,

426 current AD symptomatic therapies target acetylcholine system, by inhibiting

427 acetylcholinesterase [34]. Thus, we used human neuroblastoma SH-SY5Y cells to assess the

428 effects of NB-02 on cell viability, NGF production and acetylcholinesterase activity.

429 Cell viability was assessed using MTT assay. Exposure of SH-SY5Y cells to oligomeric Aß42

430 resulted in decreased cell survival (Fig. S3a). Treatment with donepezil, acetylcholinesterase

431 inhibitor, restored cell survival in a dose dependent manner. Interestingly, treatment with NB-02

432 restored cell survival in a dose dependent manner as well. NB-02 mediated its neuroprotective 
433 effects via serotonin receptors, since serotonin receptor 6 antagonist Lu AE58054 blocked the

434 neuroprotective effect (Fig. S3a).

435 Acetylcholinesterase activity was assessed using acetylcholinesterase activity assay. Exposure

436 of SH-SY5Y cells to oligomeric Aß42 resulted in increased acetylcholinesterase activity (Fig.

$437 \quad$ S3b). Donepezil treatment of cultures restored acetylcholinesterase activity. Also, treatment with

$438 \quad$ NB-02 restored acetylcholinesterase activity in a dose dependent manner. Its effect was

439 mediated via serotonin receptors, since Lu AE58054 blocked the effect (Fig. S3b).

440 Finally, NGF production was assessed in healthy SH-SY5Y cells, not treated with AB42. NB-02

441 increased NGF production in a dose dependent manner, while donepezil failed to do so (Fig.

442 S3c). Thus NB-02 is a multimodal therapeutic, which increased cell viability, inhibited

443 acetylcholinesterase and increased NGF production.

444 Discussion

445 NB-02, previously known as DA-9803, is an all-natural botanical drug prepared by mixing 446 natural substances that is prepared according to a standardized recipe. It has multiple modes of 447 action and has shown great promise as a preventative agent in animal models of $A D[13]$. Thus, 448 in this study we assessed its potential as a therapeutic agent.

449 Starting at 8 months of age, APP mice were treated with the therapeutic or vehicle via daily 450 gavage for two months. Amyloid plaque deposition was monitored with multiphoton microscopy 451 before, during and after treatment. As early as 1 month after treatment onset, amyloid 452 deposition was halted and some amyloid cleared in NB-02-treated mice. Animals treated with 453 vehicle continued accumulating amyloid over time. This resulted in a substantially higher 454 number of amyloid plaques in vehicle-treated animals compared to drug-treated mice at the end 455 of treatment. Amyloid burden, which considers the size of plaques as well as their number, was 456 also significantly higher in vehicle-treated mice. Interestingly, amyloid plaques appeared dimmer 
457 in NB-02 treated animals, however this was not due to NB-02 interference of methoxy-X04

458 binding to the plaques as we have reported previously[13]. Post-mortem analysis confirmed

459 elevated amyloid plaque burden in the cortices of vehicle-treated animals as well as their

460 hippocampi. This was important, since multiphoton microscopy allows assessment of amyloid in

461 a small cortical volume. Both mechanisms, decreased plaque deposition and increased plaque

462 clearance, were accountable for the difference in amyloid plaque burden between NB-02- and

463 vehicle-treated brains post-treatment. Additionally, we detected a trend toward increased

464 amyloid beta 40 and 42 levels in CSF as well as TBS-soluble fraction of the brains. However,

465 this trend failed to reach significance, possibly due to limited sensitivity of the biochemical

466 procedure. Thus, NB-02 affects extracellular plaques by preventing their deposition and aiding

467 their clearance, possibly by making amyloid more soluble and easier to clear.

468 APP mice contain a population of cortical neurons that is highly vulnerable to amyloid beta[26].

469 Not being able to maintain calcium homeostasis, these neurons exhibit calcium elevations or

470 calcium overload. As such, restoration of baseline neuronal calcium levels would serve as a

471 functional indicator of treatment efficacy[12,13,35]. Interestingly, NB-02 treatment restored

472 neuronal calcium thus leading to fewer neurons exhibiting calcium overload. Since intracellular

473 calcium supports a myriad of neuronal functions, calcium normalization would signify restoration

474 of these functions, including those supporting cognitive and memory processes of AD

475 patients[5].

476 Neuroinflammation is an integral process in AD progression[7,8]. Thus, we assessed the effect

477 of NB-02 treatment on neuroinflammation. The number of reactive astrocytes and microglia

478 were compared between conditions. However, the number of GFAP-positive astrocytes did not

479 differ significantly in vehicle vs. drug-treated mice. Similarly, IBA-1-positive microglia numbers

480 failed to reach significant difference. Analyses of morphometric changes revealed that, while

481 astrocytes maintained the small cell body and long processes in vehicle-treated animals, NB-02 
482 treatment transformed the morphology of astrocytes, allowing them to assume larger cell bodies

483 and shorter processes. Similarly, microglia morphology also changed, assuming shorter

484 processes and larger cell bodies in the NB-02 treatment condition. These morphological

485 transformations have been associated with amyloid beta phagocytosis and clearance[36].

486 In vitro studies revealed that NB-02, a botanical therapeutic mixture, is multimodal. It increased

487 cell survival and NGF production possibly by neutralizing amyloid-dependent toxicity and

488 restoring neuronal calcium homeostasis, thus restoring neuronal function. $\underline{\text { NB-02 was an }}$

489 effective acetylcholinesterase inhibitor. And finally, it upregulated neuroinflammation, further

490 potentiating amyloid clearance. Altogether, this suggests that NB-02 has great potential in the

491 clinic as a treatment for Alzheimer's disease.

492

$493 \quad$ Limitations

494 The AD animal model used here recapitulates some aspects of human disorder. Hence future 495 clinical trials of NB-02 will elucidate true clinical potential of this therapeutic.

\section{Conclusions}

498 2-month treatment with NB-02 halts plaque deposition and clears amyloid in cortex as well as

499 hippocampus. It normalizes neuronal calcium homeostasis, thus restoring neuronal function, 500 and upregulates neuroinflammation. NB-02 is a multimodal botanical therapeutic with several 501 mechanisms of action. It increases cell viability and NGF production. It also inhibits

502 acetylcholinesterase activity. The exact nature of those mechanisms will be assessed in future 503 studies.Since NB-02 was effective in slowing pathophysiology in an animal model of 504 amyloidosis, it holds great promise as a therapeutic approach to treat Alzheimer's disease. 
Declarations

Ethics approval and consent to participate

509 All the studies complied with Massachusetts General Hospital Animal Care and Use Committee

510 as well as NIH guidelines for the use of laboratory animals.

\section{Consent for publication}

512 Not applicable

\section{Availability of data and materials}

514 Data and materials are available upon request.

\section{Competing interests:}

516 The study was funded by NeuroBo Pharmaceuticals and Dong-A ST

517 Acknowledgements and funding: The research was funded by NeuroBo Pharmaceuticals,

518 Dong-A ST; the Korea Drug Development Fund funded by the Ministry of Science, Information

519 and Communication Technology; Future Planning, Ministry of Trade, Industry, and Energy, and

520 Ministry of Health and Welfare (KDDF-201606-03, Republic of Korea); and NIH R56AG060974

\section{Author contributions}

522 YFL, AL, S-Z C and ANR conducted the study, analyzed the data. ANR, BJB and KVK 523 interpreted the data. KVK wrote the manuscript. YFL, ANR and BJB edited the manuscript.

\section{Figure Legends}

525 Fig. 1 Chronic treatment with NB-02 halts deposition of cortical amyloid plaques in vivo. a 526 Experimental schematic showing the time-points of viral injections, craniotomies, multiphoton 
527 imaging sessions and gavage treatments with NB-02 or vehicle of APP mice. b, e Multiphoton

528 microscopy images of red dextran angiograms in APP mice prior to treatments with vehicle (b)

529 or NB-02 (e). c, f Multiphoton microscopy images of methoxy-X04 positive amyloid plaques in

530 cortices of APP mice prior to treatments. Note $\mathbf{b}$ and $\mathbf{c}$ were taken during the same imaging

531 session and constitute the same field of view. Also, e and $\mathbf{f}$ were taken during the same imaging

532 session and constitute the same field of view. $\mathbf{d}, \mathbf{g}$ Images of amyloid plaques taken after

533 treatments with vehicle or NB-02. Note $\mathbf{c}$ and $\mathbf{d}$ were taken during different imaging sessions

534 and constitute the same field of view. Similarly, $\mathbf{f}$ and $\mathbf{g}$ were taken during different imaging

535 sessions and constitute the same field of view. $\mathbf{h}$ Plaque numbers per cubic millimeter of cortex

536 across the two conditions over time. Statistical comparisons are made to baseline (0 months). i

537 cortical plaque densities at the end of treatment across conditions. j Amyloid plaque number

538 changes at the end of treatment compared to baseline across conditions. Vehicle treatment

539 resulted in addition of new plaques, while NB-02 treatment resulted in mild clearance. $\mathbf{k}$ Size of

540 amyloid plaques in the course of treatment across conditions. I Amyloid plaque burden, which

541 takes into account plaque number and size, over time across conditions. $\mathbf{m}$ Plaque burden at

542 the end of treatment with vehicle or NB-02. $\mathbf{n}$ Amyloid plaque burden change at the end of

543 treatment compared to baseline. o Methoxy-X04 intensity change at the end of treatment. Scale

544 bar, $100 \mu \mathrm{m}$. Mean \pm SEM. ${ }^{*} p<0.05,{ }^{* *} \mathrm{p}<0.01,{ }^{* *} \mathrm{p}<0.001$. n.s. not significant.

545 Fig. 2 NB-02 treatment results in lower cortical amyloid load compared to vehicle when

546 assessed postmortem. a, d 6E10 immunoreactivity against amyloid in brain sections obtained

547 post-mortem after treatment with vehicle or NB-02. b, e Methoxy-X04 positive amyloid plaques.

548 Images in a, b were acquired from the same field of view. Similarly, images in $\mathbf{d}$ and $\mathbf{e}$ were

549 acquired from the same field of view. c, f Colocalization of $6 \mathrm{E} 10$ and methoxy-X04. g, h Cortical

550 amyloid plaque burden as assessed by 6E10 immunoreactivity and methoxy-X04 signal. Each

551 dot represents amyloid burden in a hemibrain. Scale bar, $75 \mu \mathrm{m}$. Mean \pm SEM, ${ }^{* *} \mathrm{p}<0.01$. 
552 Fig. 3 NB-02 treatment results in lower hippocampal amyloid load compared to vehicle when assessed postmortem. a, d 6E10 immunoreactivity against amyloid in hippocampi obtained

554 post-mortem after treatment with vehicle or NB-02. b, e Methoxy-X04 positive amyloid plaques.

555 Images in a, b were taken from the same field of view. Similarly, images in $\mathbf{d}$ and $\mathbf{e}$ were taken

556 from the same field of view. c, $\mathbf{f}$ Colocalization of 6E10 and methoxy-X04. $\mathbf{g}, \mathbf{h}$ Cortical amyloid

557 plaque burden as assessed by $6 \mathrm{E} 10$ immunoreactivity and methoxy-X04 signal. Each dot

558 represents amyloid burden in a hemibrain. Scale bar, $50 \mu \mathrm{m}$. Mean \pm SEM, ${ }^{*} p<0.05,{ }^{* *} p<0.01$.

559 Fig. 4 NB-02 restores neuronal calcium overload in vivo. a, b Multiphoton microscopy images of

560 calcium reporter YC2 expressed in neurons pseudocolored according to intracellular calcium

561 concentrations in APP brains treated with vehicle (a) or NB-02 (b). Yellow arrows point to

562 neuronal cell bodies exhibiting calcium overload, while yellow arrowhead points to neuronal

563 process, neurite, exhibiting calcium overload. c, d Histograms showing percentages of neurites

564 binned into categories according to the YFP/CFP ratios over the course of treatment with

565 vehicle (c) or NB-02 (d). Percentage of neurites with calcium overload, YFP/CFP ratio>1.79, are

566 shaded in red. e Bar graph showing percentages of neurites exhibiting calcium overload at the

567 end of treatment. Scale bar, $50 \mu \mathrm{m}$. Mean \pm SEM, ${ }^{*} \mathrm{p}<0.05$.

568 Fig. 5 NB-02 transforms morphology of reactive astrocytes. a, b GFAP immunoreactivity in 569 cortical sections obtained from vehicle (a) and NB-02 (b) treated APP mice. Scale bar, $0.1 \mathrm{~mm}$.

570 c GFAP positive cell counts in cortex across conditions. d, e GFAP immunoreactivity in

571 hippocampal sections obtained from vehicle (d) and NB-02 (e) treated APP mice. Scale bar, 0.1

572 mm. f GFAP positive cell counts in hippocampus across conditions. $\mathbf{g}$, $\mathbf{h}$ Higher magnification

573 images of a single astrocyte in the brain of an APP mouse treated with vehicle (g) or NB-02 (h).

574 Scale bar, $10 \mu \mathrm{m}$. i Astrocyte process length across conditions. j Astrocyte cell body diameter

575 across conditions. n.s, not significant, ${ }^{* *} p<0.001$. 
Fig. 6 NB-02 transforms morphology of reactive microglia. a, b lba-1 immunoreactivity in cortical sections obtained from vehicle (a) and NB-02 (b) treated APP mice. Scale bar, $0.1 \mathrm{~mm}$. c lba-1 positive cell counts in cortex across conditions. d, e lba-1 immunoreactivity in hippocampal sections obtained from vehicle (d) and NB-02 (e) treated APP mice. Scale bar, $0.1 \mathrm{~mm}$. f lba-1 positive cell counts in hippocampus across conditions. $\mathbf{g}$, $\mathbf{h}$ Higher magnification images of a single microglia in the brain of an APP mouse treated with vehicle (g) or NB-02 (h). Scale bar, $10 \mu \mathrm{m}$. i Microglia process length across conditions. j Microglia cell body diameter across conditions. k Microglia soma area across conditions. I Microglia cell roundness across conditions. n.s, not significant, ${ }^{* * *} p<0.0001$.

Fig. S1 NB-02 does not alter soluble amyloid levels. a, b Human amyloid beta (a: 40, b:42) levels in cerebral spinal fluid (CSF) of APP mice treated with vehicle or NB-02. c, d Human amyloid beta (c: 40, $\mathbf{d}: 42$ ) levels in TBS-soluble brain fractions of APP mice treated with vehicle or NB-02. Each dot represents amyloid measurement in CSF or brain fraction obtained from individual mice. n.s. not significant.

Fig. S2 Cell morphology data analyzed by mouse. a Microglia process length across conditions. b Microglia soma area across conditions. c Microglia cell body diameter across conditions. d Astrocyte process length across conditions. e Astrocyte cell body diameter across conditions. ${ }^{* *} p<0.01,{ }^{* * *} p<0.001$.

Fig. S3 NB-02 has multiple mechanisms of action. a, The effect of NB-02 on cell viability of SHSY5Y under normal conditions (Nor), after application of $1 \mu \mathrm{M}$ oligomeric Aß42, after application of oligomeric $A \beta 42$ and various doses of NB-02, after application of oligomeric $A \beta 42$ and various doses of donepezil, after application of oligomeric A 342 and various doses of NB-02 and serotonin receptor 6 blocker AE. b, The effect of NB-02 on acetylcholinesterase activity in SH-SY5Y cells under normal conditions (Nor), after application of $1 \mu \mathrm{M}$ oligomeric A 342 , after application of oligomeric $A \beta 42$ and various doses of NB-02, after application of oligomeric $A \beta 42$ 
601 and various doses of donepezil, after application of oligomeric Aß42 and various doses of NB-

$602 \underline{02}$ and serotonin receptor 6 blocker AE. c, The effect of NB-02 on NGF expression in SH-SY5Y

603 cells treated with vehicle (Veh), various doses of NB-02, or donepezil. ${ }^{*} p<0.05,{ }^{* *} p<0.01,{ }^{* * *}$

$604 \quad \mathrm{p}<0.001$.

605

606

607

608

609

610

611 1. Prince M, Bryce R, Albanese E, Wimo A, Ribeiro W, Ferri CP. The global prevalence of

612 dementia: a systematic review and metaanalysis. Alzheimers Dement. United States;

$613 \quad 2013 ; 9: 63-75 . e 2$.

614 2. Holtzman DM, Goate A, Kelly J, Sperling R. Mapping the road forward in Alzheimer's

615 disease. Sci Transl Med. 2011/12/23. 2011;3:114ps48.

616 3. Selkoe DJ. Cell biology of the amyloid beta-protein precursor and the mechanism of

617 Alzheimer's disease. Annu Rev Cell Biol. 1994/01/01. 1994;10:373-403.

618 4. Hardy J, Selkoe DJ. The amyloid hypothesis of Alzheimer's disease: progress and problems 619 on the road to therapeutics. Science (80- ). 2002/07/20. 2002;297:353-6.

620 5. Khachaturian ZS. Calcium hypothesis of Alzheimer's disease and brain aging. Ann N Y Acad 621 Sci. 1994/12/15. 1994;747:1-11.

622 6. Selkoe DJ. Alzheimer's disease is a synaptic failure. Science (80- ). 2002/10/26.

$623 \quad 2002 ; 298: 789-91$.

624 7. Frost GR, Li Y-M. The role of astrocytes in amyloid production and Alzheimer's disease. 
626 8. Gonzalez-Reyes RE, Nava-Mesa MO, Vargas-Sanchez K, Ariza-Salamanca D, Mora-Munoz

627 L. Involvement of Astrocytes in Alzheimer's Disease from a Neuroinflammatory and Oxidative

628 Stress Perspective. Front Mol Neurosci. Switzerland; 2017;10:427.

629 9. Frost GR, Li Y, Frost GR. The role of astrocytes in amyloid production and Alzheimer's 630 disease. Open Biol. 2017;7:1-14.

631 10. Heneka MT, Carson MJ, El Khoury J, Landreth GE, Brosseron F, Feinstein DL, et al.

632 Neuroinflammation in Alzheimer's disease. Lancet Neurol. England; 2015;14:388-405.

633 11. Kastanenka K V., Bussiere T, Shakerdge N, Qian F, Weinreb PH, Rhodes K, et al. 634 Immunotherapy with Aducanumab Restores Calcium Homeostasis in Tg2576 Mice. J Neurosci 635 [Internet]. 2016 [cited 2017 Feb 1];36:12549-58. Available from:

636 http://www.ncbi.nlm.nih.gov/pubmed/27810931

637 12. Wang X, Kastanenka KV, Arbel-Ornath M, Commins C, Kuzuya A, Lariviere AJ, et al. An 638 acute functional screen identifies an effective antibody targeting amyloid- $\beta$ oligomers based on 639 calcium imaging. Sci Rep. 2018;8.

640 13. Pagnier GJ, Kastanenka K V, Sohn M, Choi S, Choi S-H, Soh H, et al. Novel botanical drug 641 DA-9803 prevents deficits in Alzheimer's mouse models. Alzheimers Res Ther. England; $642 \quad 2018 ; 10: 11$.

643 14. Jankowsky JL, Fadale DJ, Anderson J, Xu GM, Gonzales V, Jenkins NA, et al. Mutant 644 presenilins specifically elevate the levels of the 42 residue beta-amyloid peptide in vivo:

645 evidence for augmentation of a 42-specific gamma secretase. Hum Mol Genet. England; $646 \quad 2004 ; 13: 159-70$.

647 15. Levasseur JE, Wei EP, Raper AJ, Kontos AA, Patterson JL. Detailed description of a cranial 
648

649

650

651

652

653

654

655

656

657

658

659

660

661

662

663

664

665

666

667

668

669

670

window technique for acute and chronic experiments. Stroke. United States; 1975;6:308-17.

16. Kastanenka K V, Hou SS, Shakerdge N, Logan R, Feng D, Wegmann S, et al. Optogenetic Restoration of Disrupted Slow Oscillations Halts Amyloid Deposition and Restores Calcium Homeostasis in an Animal Model of Alzheimer's Disease. Ginsberg SD, editor. PLoS One [Internet]. 2017 [cited 2017 Feb 1];12:e0170275. Available from:

http://dx.plos.org/10.1371/journal.pone.0170275

17. Wang X, Kastanenka K V, Arbel-Ornath M, Commins C, Kuzuya A, Lariviere AJ, et al. An acute functional screen identifies an effective antibody targeting amyloid-beta oligomers based on calcium imaging. Sci Rep. England; 2018;8:4634.

18. Nagai T, Yamada S, Tominaga T, Ichikawa M, Miyawaki A. Expanded dynamic range of fluorescent indicators for $\mathrm{Ca}(2+)$ by circularly permuted yellow fluorescent proteins. Proc Natl Acad Sci U S A [Internet]. 2004;101:10554-9. Available from:

http://www.ncbi.nlm.nih.gov/pubmed/15247428

19. Klunk WE, Bacskai BJ, Mathis CA, Kajdasz ST, McLellan ME, Frosch MP, et al. Imaging Abeta plaques in living transgenic mice with multiphoton microscopy and methoxy-X04, a systemically administered Congo red derivative. J Neuropathol Exp Neurol. 2002/09/17. 2002;61:797-805.

20. Bacskai BJ, Klunk WE, Mathis CA, Hyman BT. Imaging amyloid-beta deposits in vivo. J Cereb Blood Flow Metab. 2002/09/10. 2002;22:1035-41.

21. Garcia-Alloza M, Robbins EM, Zhang-Nunes SX, Purcell SM, Betensky RA, Raju S, et al. Characterization of amyloid deposition in the APPswe/PS1dE9 mouse model of Alzheimer disease. Neurobiol Dis [Internet]. 2006;24:516-24. Available from:

http://www.ncbi.nlm.nih.gov/pubmed/17029828 
671

672 $673 \quad 1961 ; 7: 88-95$.

674

675

676

677

678

679

680

681

682

683

684

685

686

687

688

689

690

691

692

693 Group; 2015.

22. ELLMAN GL, COURTNEY KD, ANDRES VJ, FEATHER-STONE RM. A new and rapid colorimetric determination of acetylcholinesterase activity. Biochem Pharmacol. England;

23. Kastanenka K V, Arbel-Ornath M, Hudry E, Galea E, Xie H, Bacskai BJ. Optical probes for in-vivo imaging. In: Zhang J, Schultz C, editors. Opt Probes Biol. USA: Taylor and Francis

24. Bacskai BJ, Hyman BT. Alzheimer's disease: what multiphoton microscopy teaches us. Neuroscientist. 2002/10/11. 2002;8:386-90.

25. Wang X, Arbel-Ornath M, Wegmann SK, Kastanenka KV, Bacskai BJ. In vivo imaging in neurodegenerative diseases. Adv. Intravital Microsc. From Basic to Clin. Res. 2014.

26. Kuchibhotla K V, Goldman ST, Lattarulo CR, Wu HY, Hyman BT, Bacskai BJ. Abeta plaques lead to aberrant regulation of calcium homeostasis in vivo resulting in structural and functional disruption of neuronal networks. Neuron [Internet]. 2008;59:214-25. Available from: http://www.ncbi.nlm.nih.gov/pubmed/18667150

27. Holtzman DM, Morris JC, Goate AM. Alzheimer's disease: the challenge of the second century. Sci Transl Med. 2011/04/08. 2011;3:77sr1.

28. Akiyama H, Barger S, Barnum S, Bradt B, Bauer J, Cole GM, et al. Inflammation and Alzheimer's disease. Neurobiol Aging. United States; 2000;21:383-421.

29. Wyss-Coray T. Inflammation in Alzheimer disease: driving force, bystander or beneficial response? Nat Med. United States; 2006;12:1005-15.

30. Eng LF, Ghirnikar RS. GFAP and astrogliosis. Brain Pathol. Switzerland; 1994;4:229-37.

31. Mucke L, Oldstone MB, Morris JC, Nerenberg MI. Rapid activation of astrocyte-specific expression of GFAP-lacZ transgene by focal injury. New Biol. United States; 1991;3:465-74. 
694 32. Counts SE, Mufson EJ. The role of nerve growth factor receptors in cholinergic basal

695 forebrain degeneration in prodromal Alzheimer disease. J Neuropathol Exp Neurol. England;

$696 \quad 2005 ; 64: 263-72$.

697 33. Luo JJ, Wallace MS, Hawver DB, Kusiak JW, Wallace WC. Characterization of the 698 neurotrophic interaction between nerve growth factor and secreted alpha-amyloid precursor 699 protein. J Neurosci Res. United States; 2001;63:410-20.

700 34. Singh M, Kaur M, Kukreja H, Chugh R, Silakari O, Singh D. Acetylcholinesterase inhibitors 701 as Alzheimer therapy: from nerve toxins to neuroprotection. Eur J Med Chem. France; $702 \quad 2013 ; 70: 165-88$.

703 35. Kastanenka K V., Bussiere T, Shakerdge N, Qian F, Weinreb PH, Rhodes K, et al. 704 Immunotherapy with Aducanumab Restores Calcium Homeostasis in Tg2576 Mice. J Neurosci 705 [Internet]. 2016;36:12549-58. Available from:

706 http://www.jneurosci.org/lookup/doi/10.1523/JNEUROSCI.2080-16.2016

707 36. laccarino HF, Singer AC, Martorell AJ, Rudenko A, Gao F, Gillingham TZ, et al. Gamma 708 frequency entrainment attenuates amyloid load and modifies microglia. Nature [Internet]. 709 2016;540:230-5. Available from: http://www.nature.com/doifinder/10.1038/nature20587 


\section{Figures}
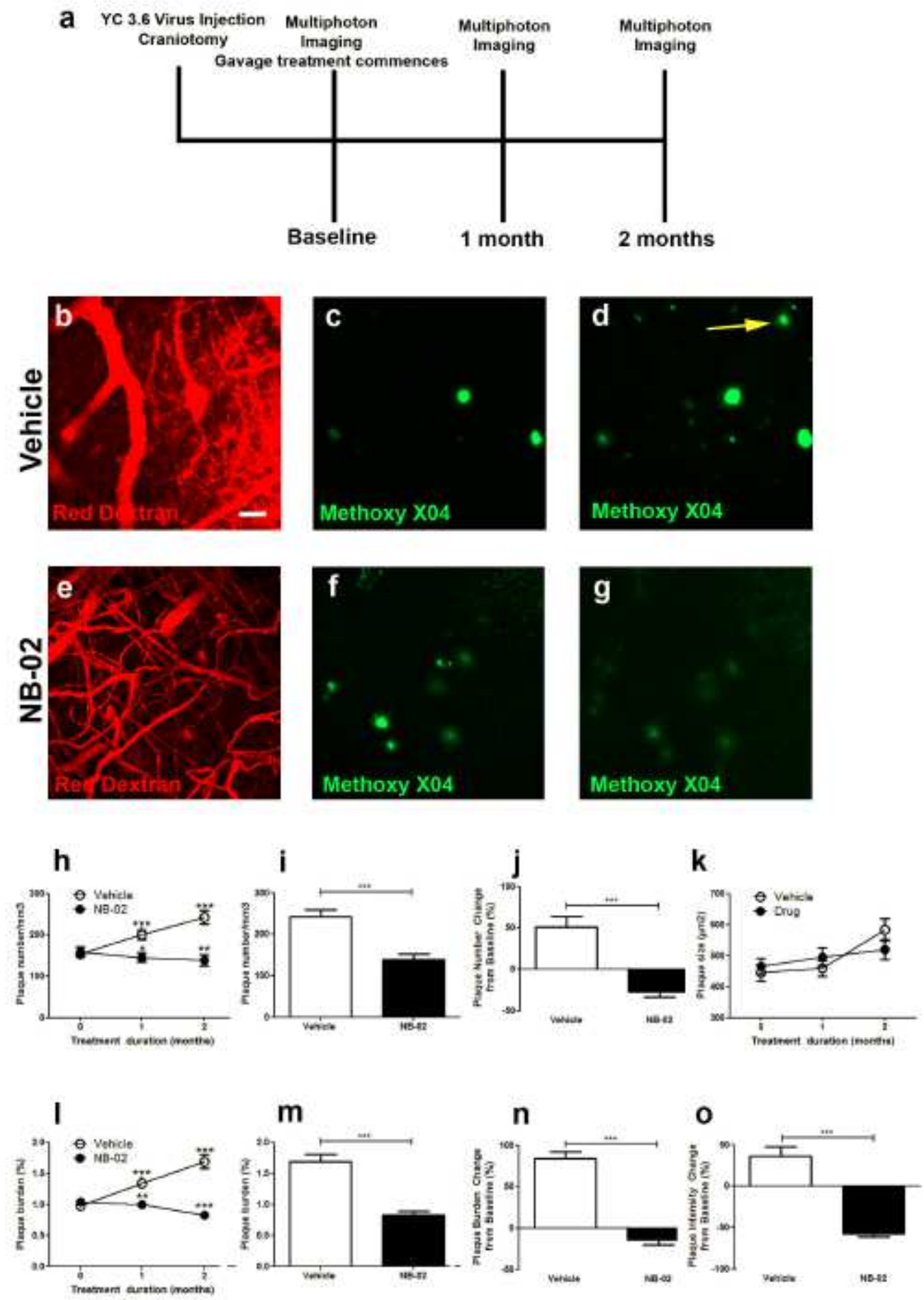

\section{Figure 1}

Chronic treatment with NB-02 halts deposition of cortical amyloid plaques in vivo. a Experimental schematic showing the time-points of viral injections, craniotomies, multiphoton imaging sessions and gavage treatments with NB-02 or vehicle of APP mice. b, e Multiphoton microscopy images of red dextran angiograms in APP mice prior to treatments with vehicle (b) or NB-02 (e). c, f Multiphoton microscopy 
images of methoxy-X04 positive amyloid plaques in cortices of APP mice prior to treatments. Note $b$ and $\mathrm{c}$ were taken during the same imaging session and constitute the same field of view. Also, $e$ and $f$ were taken during the same imaging session and constitute the same field of view. $\mathrm{d}, \mathrm{g}$ Images of amyloid plaques taken after treatments with vehicle or NB-02. Note $\mathrm{c}$ and $\mathrm{d}$ were taken during different imaging sessions and constitute the same field of view. Similarly, $f$ and $g$ were taken during different imaging sessions and constitute the same field of view. h Plaque numbers per cubic millimeter of cortex across the two conditions over time. Statistical comparisons are made to baseline ( 0 months). i cortical plaque densities at the end of treatment across conditions. j Amyloid plaque number changes at the end of treatment compared to baseline across conditions. Vehicle treatment resulted in addition of new plaques, while NB-02 treatment resulted in mild clearance. $\mathrm{k}$ Size of amyloid plaques in the course of treatment across conditions. I Amyloid plaque burden, which takes into account plaque number and size, over time across conditions. $m$ Plaque burden at the end of treatment with vehicle or NB-02. $n$ Amyloid plaque burden change at the end of treatment compared to baseline. o Methoxy-X04 intensity change at the end of treatment. Scale bar, $100 \mu$ m. Mean \pm SEM. ${ }^{\star} p<0.05,{ }^{*} p<<0.01$, ${ }^{\star \star *} p<0.001$. n.s. not significant.
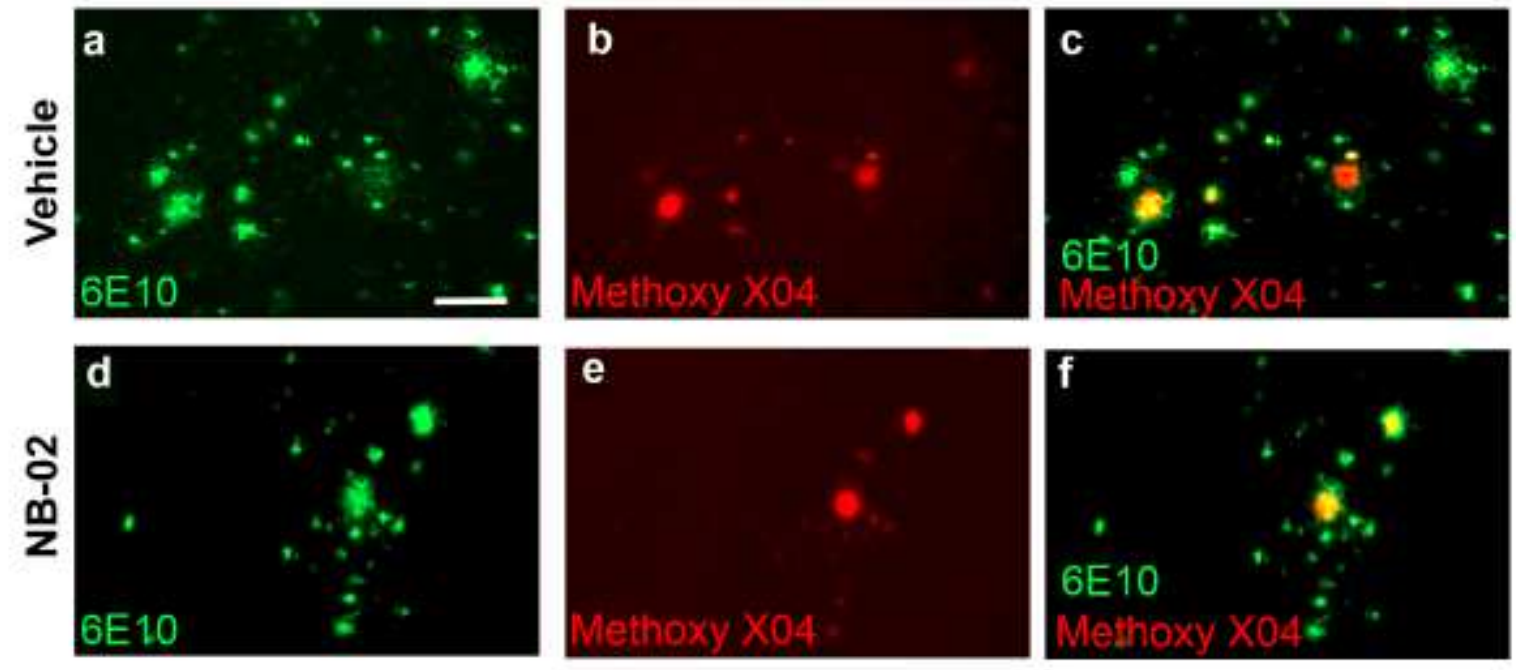

g

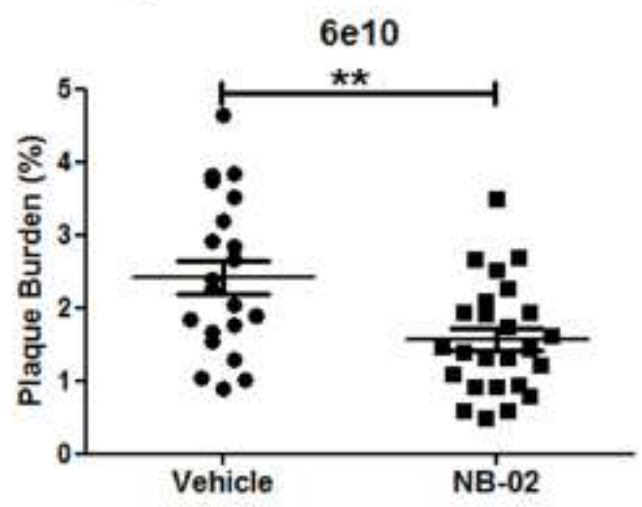

h

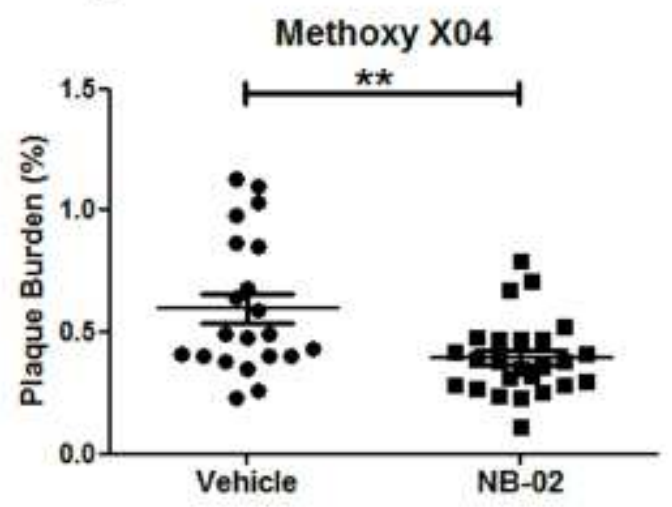

Figure 2 
NB-02 treatment results in lower cortical amyloid load compared to vehicle when assessed postmortem. a, d 6E10 immunoreactivity against amyloid in brain sections obtained post-mortem after treatment with vehicle or NB-02. b, e Methoxy-X04 positive amyloid plaques. Images in a, b were acquired from the same field of view. Similarly, images in $d$ and e were acquired from the same field of view. $c, f$ Colocalization of 6E10 and methoxy-X04. $\mathrm{g}$, h Cortical amyloid plaque burden as assessed by $6 \mathrm{E} 10$ immunoreactivity and methoxy-X04 signal. Each dot represents amyloid burden in a hemibrain. Scale bar, $75 \mu \mathrm{m}$. Mean $\pm \mathrm{SEM}$, $\star * p<0.01$.
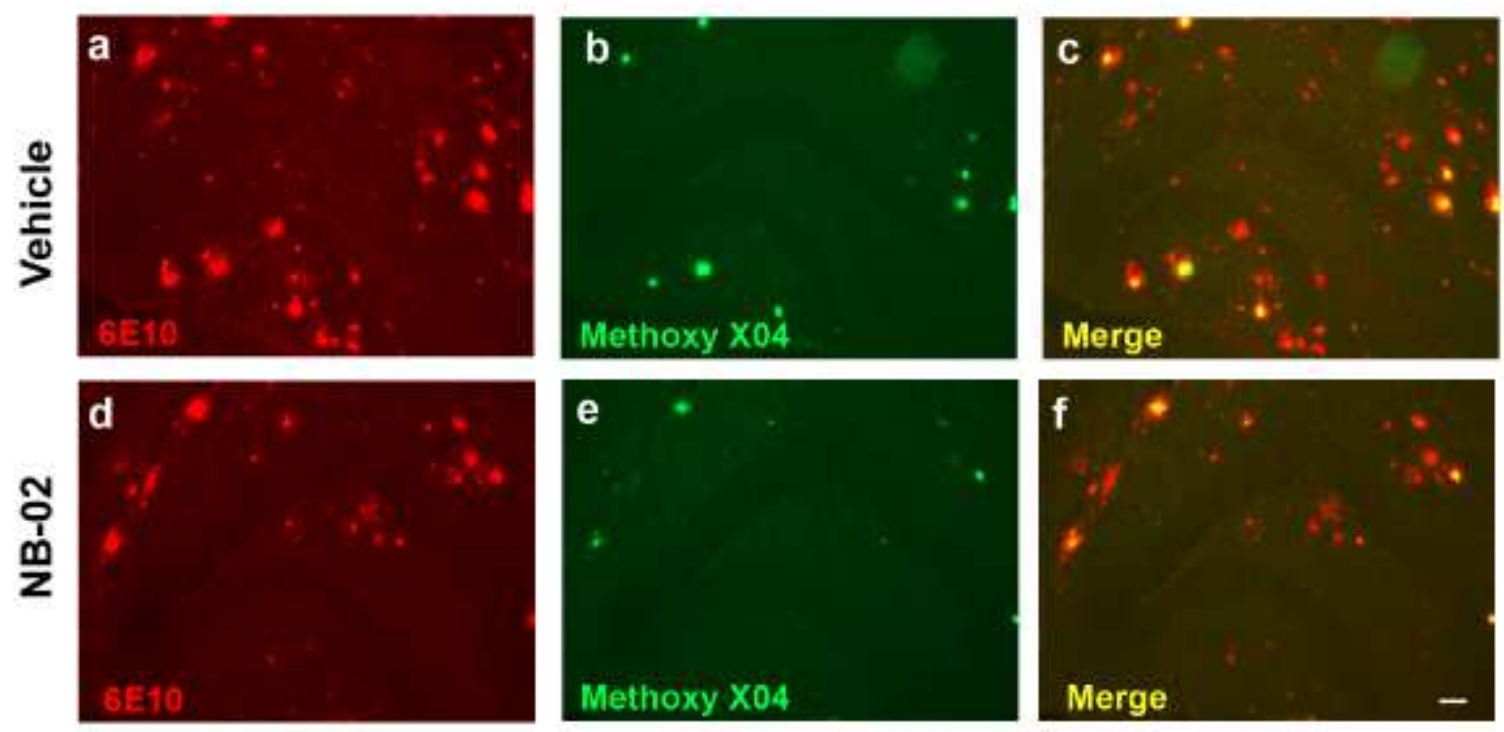

g

h
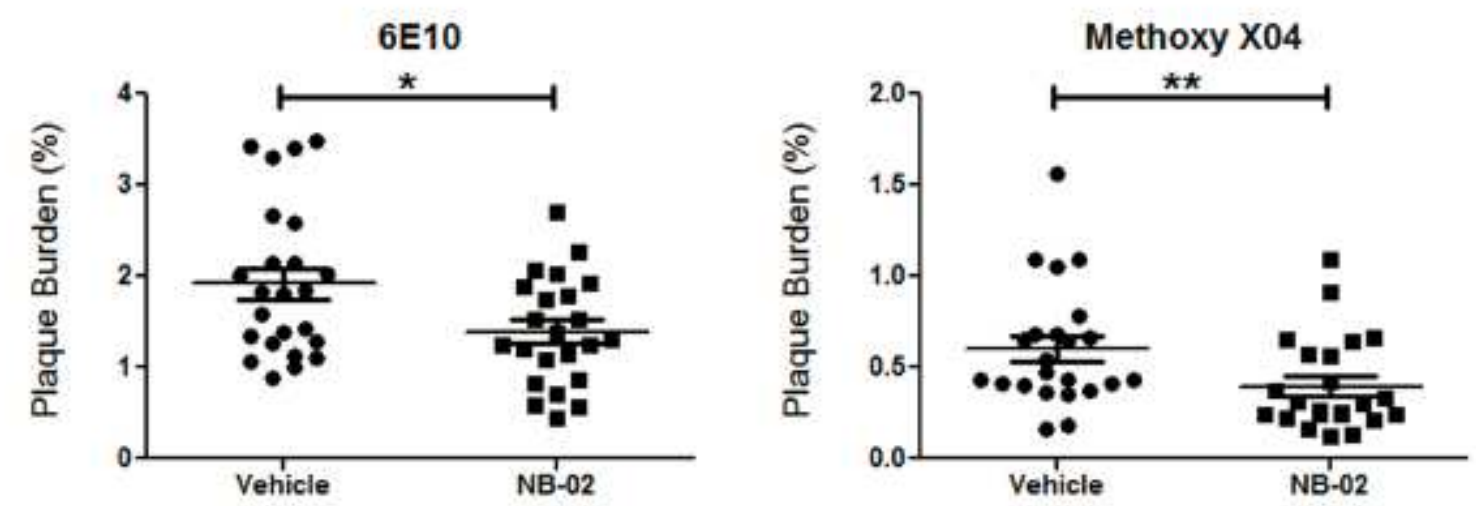

Figure 3

NB-02 treatment results in lower hippocampal amyloid load compared to vehicle when assessed postmortem. a, d 6E10 immunoreactivity against amyloid in hippocampi obtained post-mortem after treatment with vehicle or NB-02. b, e Methoxy-X04 positive amyloid plaques. Images in a, b were taken from the same field of view. Similarly, images in $d$ and e were taken from the same field of view. $c, f$ Colocalization of 6E10 and methoxy-X04. g, h Cortical amyloid plaque burden as assessed by 6E10 immunoreactivity and methoxy-X04 signal. Each dot represents amyloid burden in a hemibrain. Scale bar, $50 \mu \mathrm{m}$. Mean \pm SEM, ${ }^{\star} \mathrm{p}<0.05,{ }^{\star *} \mathrm{p}<0.01$. 


\section{a

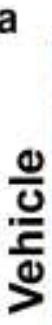

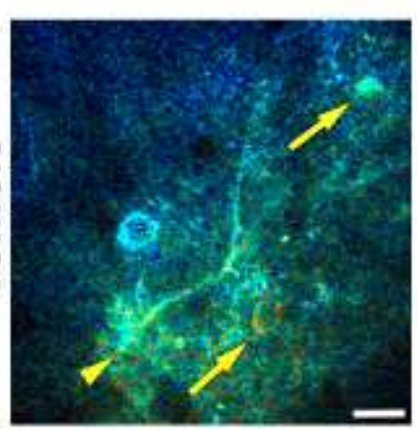
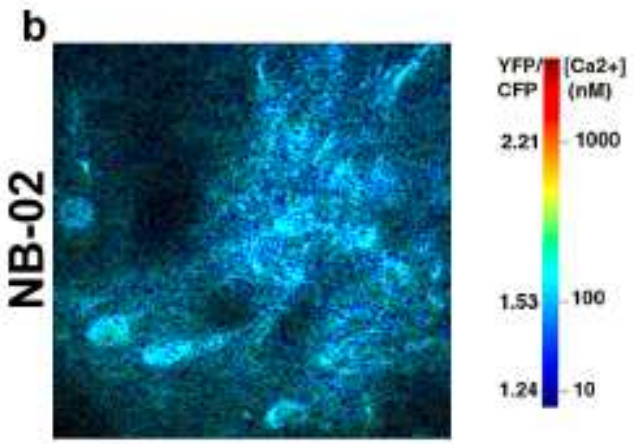

C

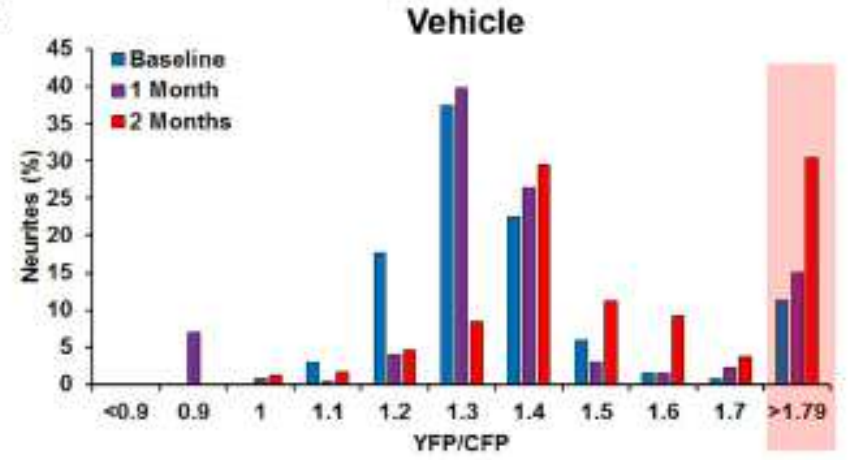

d

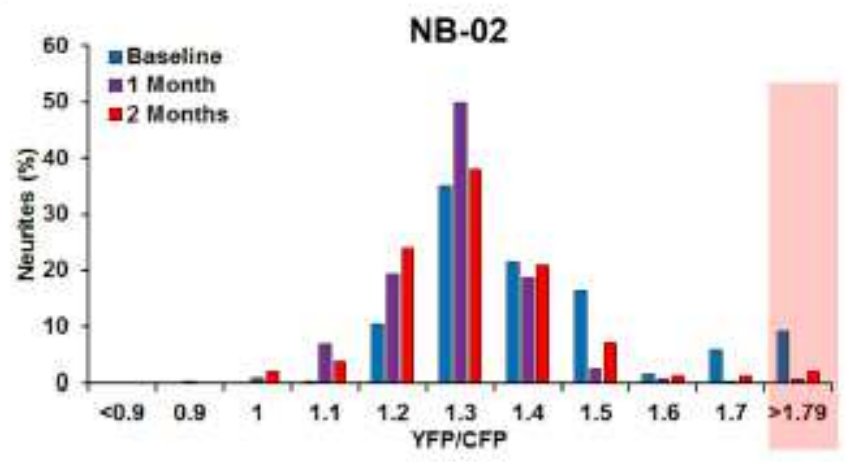

e

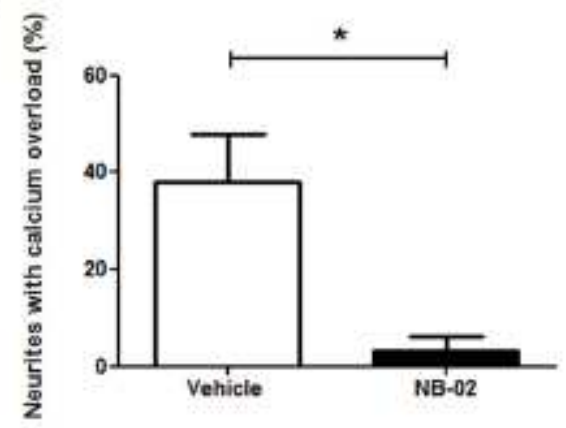

Figure 4

NB-02 restores neuronal calcium overload in vivo. a, b Multiphoton microscopy images of calcium reporter YC2 expressed in neurons pseudocolored according to intracellular calcium concentrations in APP brains treated with vehicle (a) or NB-02 (b). Yellow arrows point to neuronal cell bodies exhibiting calcium overload, while yellow arrowhead points to neuronal process, neurite, exhibiting calcium overload. c, d Histograms showing percentages of neurites binned into categories according to the 
YFP/CFP ratios over the course of treatment with vehicle (c) or NB-02 (d). Percentage of neurites with calcium overload, YFP/CFP ratio $>1.79$, are shaded in red. e Bar graph showing percentages of neurites exhibiting calcium overload at the end of treatment. Scale bar, $50 \mu \mathrm{m}$. Mean $\pm \mathrm{SEM},{ }^{*} \mathrm{p}<0.05$.

\section{Vehicle}
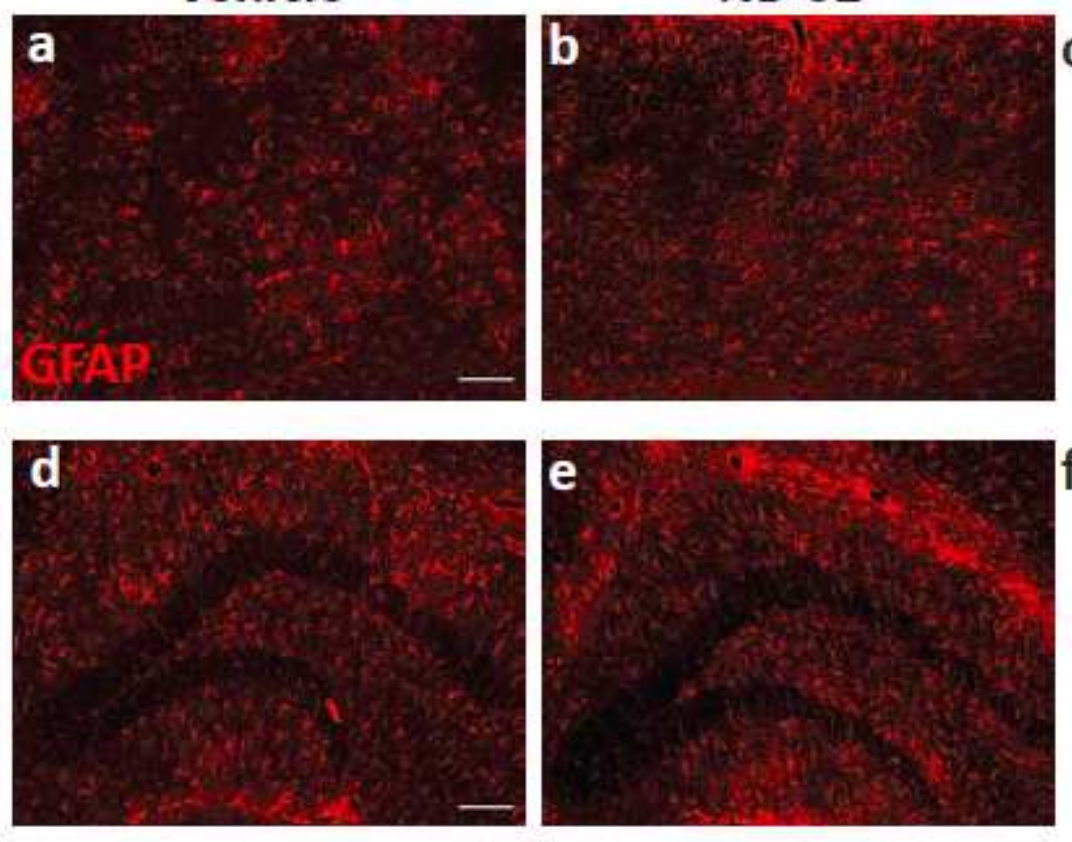

NB-02
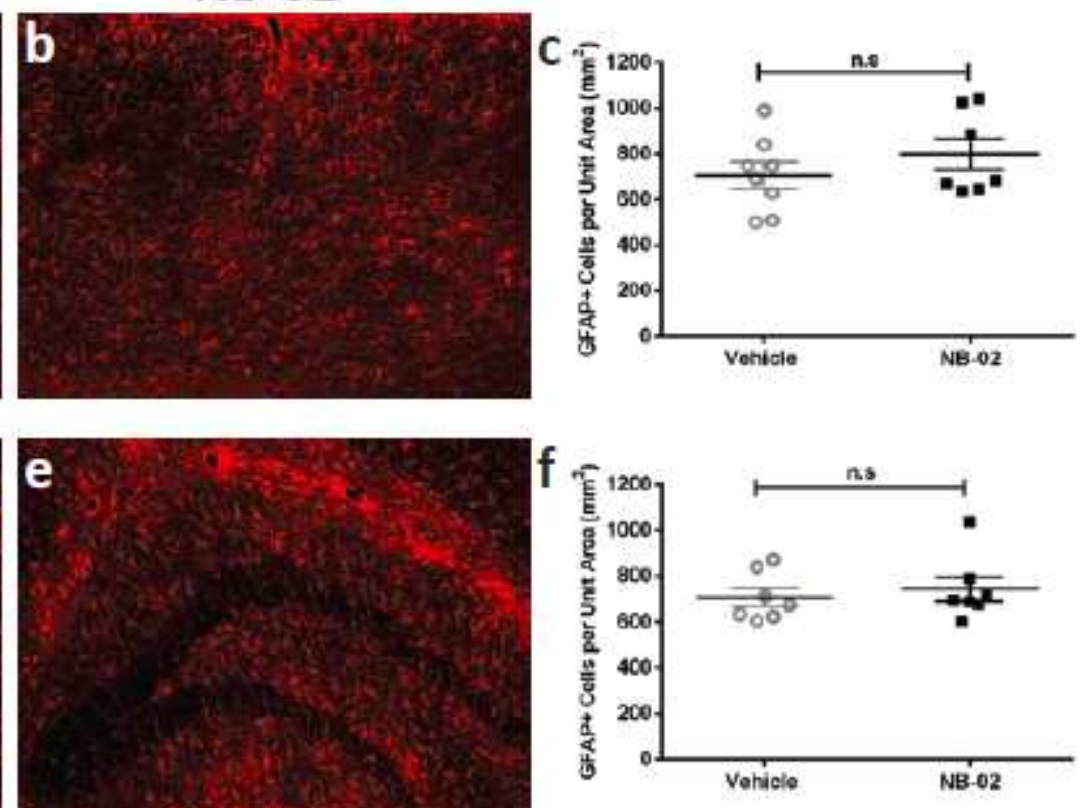

\section{Vehicle}

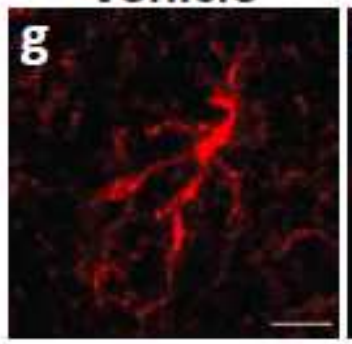

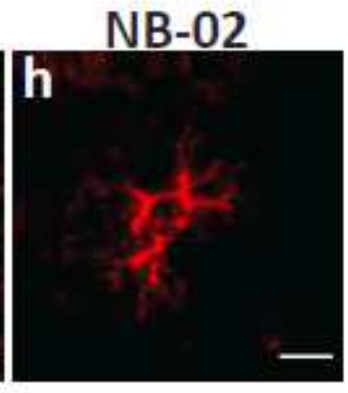
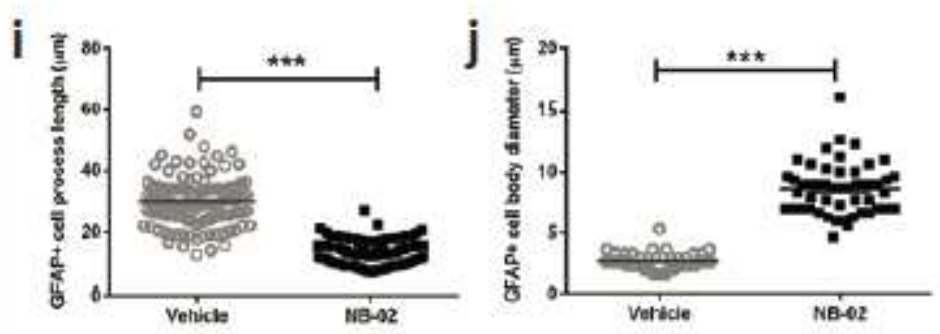

Figure 5

NB-02 transforms morphology of reactive astrocytes. a, b GFAP immunoreactivity in cortical sections obtained from vehicle (a) and NB-02 (b) treated APP mice. Scale bar, $0.1 \mathrm{~mm}$. c GFAP positive cell counts in cortex across conditions. d, e GFAP immunoreactivity in hippocampal sections obtained from vehicle (d) and NB-02 (e) treated APP mice. Scale bar, $0.1 \mathrm{~mm}$. f GFAP positive cell counts in hippocampus across conditions. $\mathrm{g}$, h Higher magnification images of a single astrocyte in the brain of an APP mouse treated with vehicle $(\mathrm{g})$ or NB-02 (h). Scale bar, $10 \mu \mathrm{m}$. i Astrocyte process length across conditions. $\mathrm{j}$ Astrocyte cell body diameter across conditions. n.s, not significant, ${ }^{\star \star \star} p<0.001$. 
Vehicle
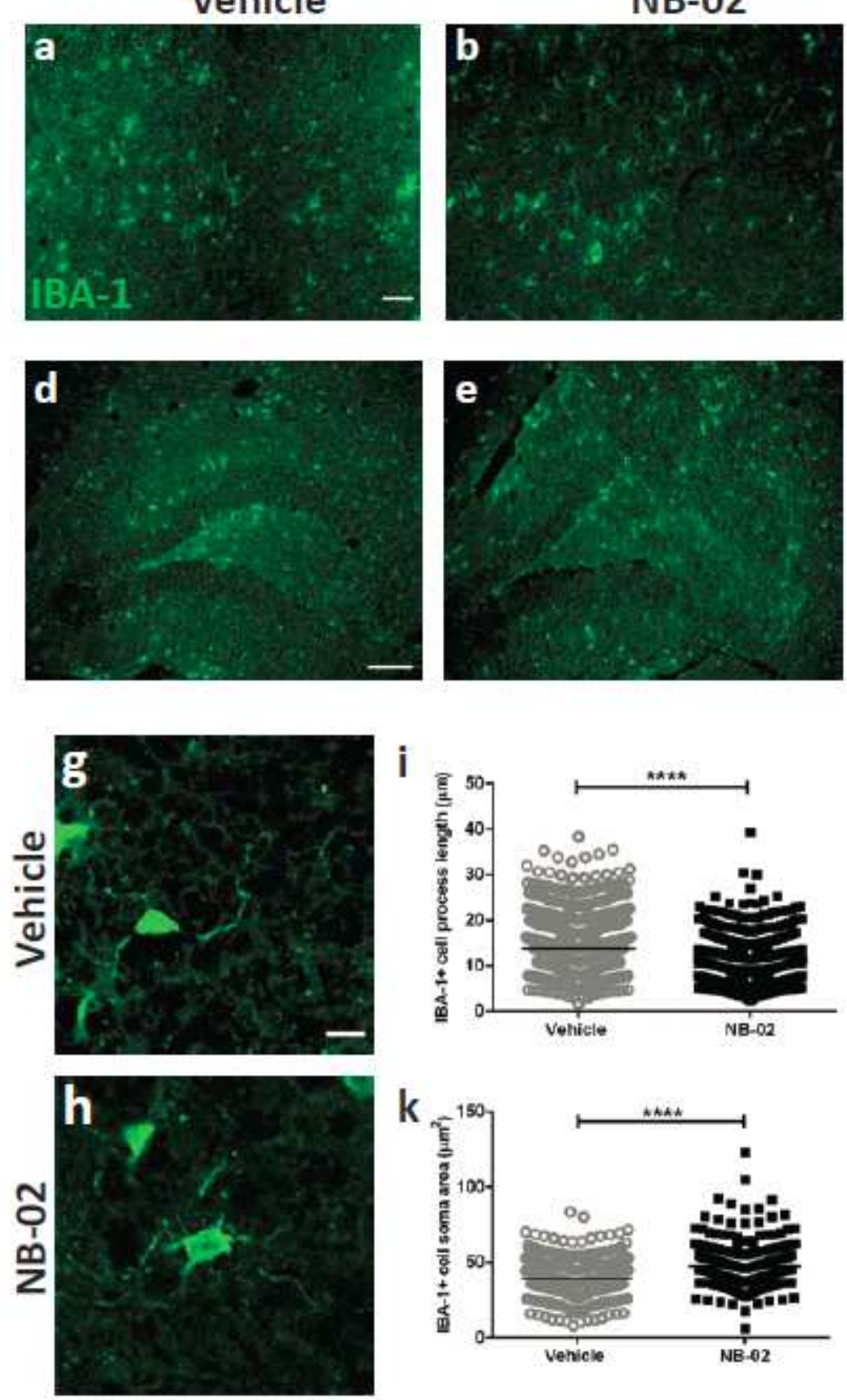
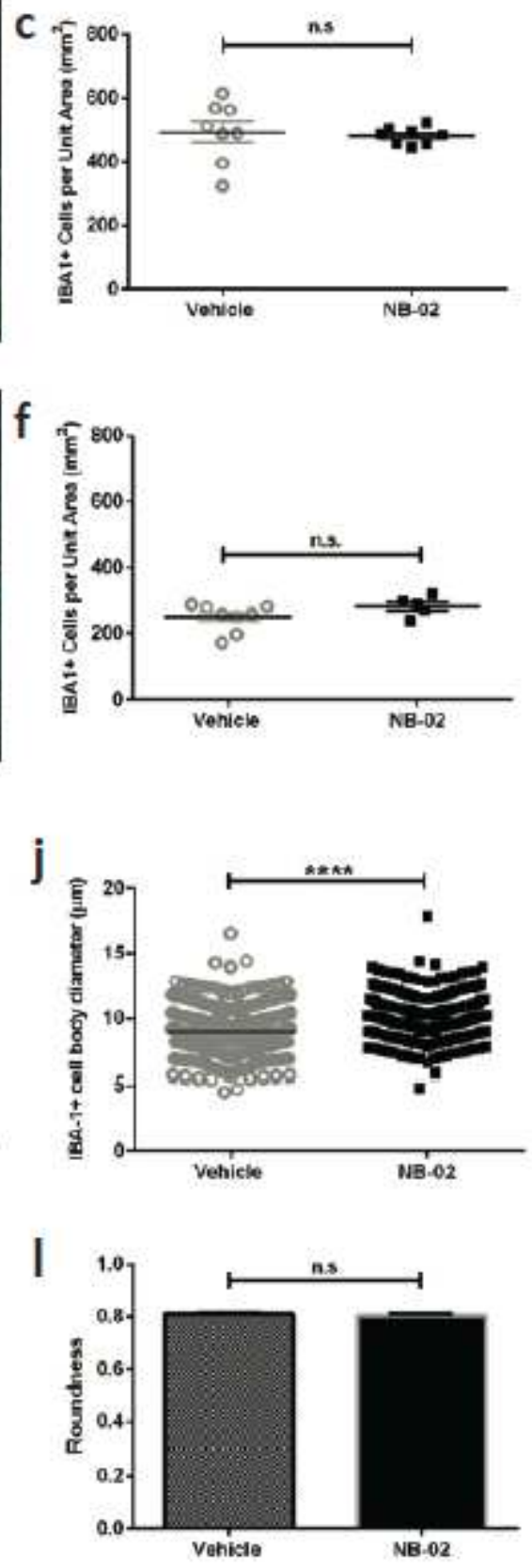

Figure 6

NB-02 transforms morphology of reactive microglia. a, b lba-1 immunoreactivity in cortical sections obtained from vehicle (a) and NB-02 (b) treated APP mice. Scale bar, $0.1 \mathrm{~mm}$. c lba-1 positive cell counts in cortex across conditions. d, e lba-1 immunoreactivity in hippocampal sections obtained from vehicle (d) and NB-02 (e) treated APP mice. Scale bar, $0.1 \mathrm{~mm}$. $\mathrm{f} \mathrm{Iba-1}$ positive cell counts in hippocampus across conditions. $g$, h Higher magnification images of a single microglia in the brain of an APP mouse treated with vehicle (g) or NB-02 (h). Scale bar, $10 \mu \mathrm{m}$. i Microglia process length across conditions. j Microglia cell body diameter across conditions. $\mathrm{k}$ Microglia soma area across conditions. I Microglia cell roundness across conditions. n.s, not significant, $* \star \star \star ~ p<0.0001$. 


\section{Supplementary Files}

This is a list of supplementary files associated with this preprint. Click to download.

- SupplementaryInformation.pdf 